\title{
Chronic venous disorders: from macrocirculation to microcirculation
}

\section{PL Antignani ${ }^{1}$}

${ }^{1}$ Director, Vascular Center, Nuova Villa Claudia, Rome, Italy

submitted: Jun 25, 2018, accepted: Jun 25, 2018, EPub Ahead of Print: Jun 26, 2018, published: Jun 30, 2018

Conflict of interest: None

DOI: 10.24019/jtavr.49 - Corresponding author: Prof. Pier Luigi Antignani, antignanipl@gmail.com

(C) 2017 Fondazione Vasculab impresa sociale ONLUS. All rights reserved.

Recent findings suggest that inflammatory processes are involved in the structural remodelling in venous valves and in the vein wall, leading to valve incompetence and the development of varicose veins. This has been shown by several authors who found infiltration of valve leaflets and the venous wall by leukocytes (monocytes and tissue macrophages) in all valve specimens from patients with chronic venous disease (CVD) and in none from controls ${ }^{1,2}$.

Vein wall remodelling is likely to involve the complex interplay of a range of factors, including an altered ratio between metalloproteinases (MMPs), particularly MMP9, and their tissue inhibitors (TIMPs), and elevated levels of cytokines and growth factors favour an alteration of the extracellular matrix. Neutrophils and mast cells and their interaction with the venous endothelium are believed to play an important role in the initiation of the inflammatory response in $\mathrm{CVD}^{3,4}$.

The transmission of high venous pressures to the dermal microcirculation results in the stimulation of an inflammatory process in which cytokine and growth factor release leads to leukocyte migration into the interstitium and the initiation of further inflammatory events. This process is associated with the intense dermal fibrosis and tissue remodelling seen in chronic venous insufficiency ${ }^{5}$.

The many manifestations of the disease are frequently associated with symptoms usually ascribed to CVD. The proportion of patients with symptoms increases with increasing CEAP clinical classes, but the mechanisms underlying symptom appearance have not been elucidated. It has been postulated that it is related to the inflammatory cascade of events seen at all stages of CVD and in which the leukocyte and its interaction with the endothelium play a key role. It is increasingly believed that the emerging twin themes of disturbed venous flow patterns and chronic inflammation underlie and link all the manifestations of the disease $^{6}$.

A potentially confusing aspect in clinical practice which still needs to be clarified concerns the patient's perception of symptoms (such as heaviness and pain) which according to some studies, could be confined to the superficial venous circulation, where it is estimated that over $90 \%$ of the $\mathrm{C}$ nociceptive fibers of the entire venous system is concentrated ${ }^{6}$.

Some genetic, environmental and behavioral risk factors (family history, seasonality and orthostatism) cause an increase in hydrostatic pressure, leading to valve dysfunction and the resulting venous reflux.

The increase in venous pressure also increases the tension of the endothelial wall, which stimulates the production and activity of MMPs. Increased venous pressure also causes loss of endoluminal glycosaminoglycans (GAGs glycocalyx), endothelial cell lesions, resulting in increased vascular permeability, leukocyte infiltration and increased adhesion molecules (ICAM-1, VCAM-1, L- selectin, P-selectin) inflammatory cytokines and reactive oxygen species (ROS) with the end result of a further increase in MMPs ${ }^{7,8}$.

Venous wall distension may also result in the increase of other MMPs inducers, such as EMMPRIN (extracellular matrix metalloproteinase inducer), hormones and NGAL (neutrophil gelatinase-associated lipocalin $)^{4}$. High levels 
of MMPs activate specific protease receptors on endothelial cells, leading to a reduced endothelial production of Nitric Oxide (NO), which inhibits VSM contraction and stimulates venous dilatation. MMPs also stimulate endothelial cells to produce EDHF (the hyperpolarizing factor of endothelial derivation), which hyperpolarizes cell membranes, with further loss of VSM's contractile function and the consequent amplification of the venous relaxation. The activity of the MMPs further supports venous dilatation through the release of growth factors (such as TGF beta, VGF, FGF), which stimulate VSM hypertrophy. The hyperactivity of the MMPs finally degrades the ECM, triggering a mechanism of migration of the SMC, with further dilation of the venous wall and the consequent establishment of varicose veins ${ }^{3,4}$.

Increase in MMPs can also cause hyperpolarization and relaxation of the smooth muscle cells (VSM) and ECM degradation, leading to dilatation of the venous wall, valve dysfunction and to the progressive increase of venous hydrostatic pressure, thus establishing, an actual vicious circle of hemodynamic and inflammatory self-feeding. The formation of varicose veins and their tortuosity is also favored by the action of MMPs, which degrade the extracellular matrix in particular in the atrophic regions ${ }^{5-8}$.

The structure and function of the venous wall are regulated by a series of metabolic activities involving ions, molecules and enzymes. Matrix metalloproteinases (MMPs) are Zinc (Zn) dependent endopeptidases, widely known for their ability to degrade various extracellular matrix (ECM) proteins. For this reason, MMPs play a central role in remodelling the venous tissue, degrading various components of the matrix.

MMPs can interact with bioactive molecules on the cell membrane and regulate cellular receptors and signaling, influencing cell proliferation, migration and differentiation, thus being directly involved also in cell apoptosis, immune response, tissue repair and in angiogenesis ${ }^{6,7}$.

\section{References}

1) Saharay M, Shields DA, Porter JB, Scurr JH, Coleridge-Smith PD. Leucocyte activity in the microcirculation in patients with chronic venous disease. J Vasc Surg 1997;26:265-273.

2) Antignani PL. News in inflammatory and microcirculatory mechanisms in venous ulcers. Allegra C, Antignani PL, Kalodiki E, editors. In: News in Phlebology. Minerva Medica. 2013; 211-214.

3) Kucukguven A, Khalil RA. Matrix metalloproteinases as potential targets in the venous dilation associated with varicose veins. Curr Drug Targets. 2013;14(3):287-324.

4) Chen Y, Peng W, Raffetto JD, Khalil RA. Matrix Metalloproteinases in Remodeling of Lower Extremity Veins and Chronic Venous Disease. Prog Mol Biol Transl Sci. 2017;147:267-299.
The venous smooth muscle cells (SMC) appear disorganized in the media and the adjacent intima tunica, with abundant unstructured and undefined material. Furthermore, collagen fibers appear disorganized, making it difficult to distinguish the media tunica from the external one, and the elastic fibers appear thick and fragmented. The VV show an imbalance in the ECM protein components mainly due to changes in the collagen and/or elastin content in the venous walls. Experiments on SMC in VV culture and fibroblasts cultured from patients with CVD showed an increase in the synthesis of type I collagen and a decrease in the synthesis of type III collagen. Type III collagen is a critical factor in determining the elasticity and distensibility of blood vessels and the alterations in the collagen synthesis and the type I / type III relationship could cause alterations in the integrity of the venous wall, resulting in structural weakness, dilatation and formation of $\mathrm{VVs}^{5,6}$.

This pathological process can be physiologically counterbalanced by a compensatory anti-inflammatory response system which involves prostaglandins and their receptors leading to decreased MMPs and therefore to an accumulation of ECM, particularly in the hypertrophic regions of varicose veins ${ }^{6}$.

Among the many pathophysiologic mechanisms at work, the leukocyte-endothelium interactions seem to be important in many aspects of the disease and have been identified as a possible target for pharmacologic intervention. Pharmacologic agents that could attenuate various elements of the inflammatory cascade and inhibit the inflammatory process might offer a greater opportunity to prevent future morbidity 6,7 . It seems reasonable to speculate that such treatment could reduce the risk of CVD progression if applied as soon as the first symptoms appear.

\section{Pier Luigi Antignani}

Rome

5) Shoab SS, Scurr JH, Coleridge-Smith PD. Increased plasma vascular endothelial growth factor among patients with chronic venous disease. J Vasc Surg 1998;28:535-540.

6) Antignani PL. Medical Treatment of Chronic Venous Disease. SM J Pharmac Ther. 2017;3(1):1015.

7) Castro-Ferreira R, Cardoso R, Leite-Moreira A, Mansilha A. The role of endothelial dysfunction and inflammation in chronic venous disease. Ann Vasc Surg. 2018;46:380-393.

8) Apollonio A, Antignani PL, Di Salvo M, Failla G, Guarnera G, Mosti G, Ricci E; SUV Study Group. A large Italian observational multicentre study on vascular ulcers of the lower limbs. Int Wound J. 2016;13(1):27-34. 